\title{
Liver transplantation for non-resectable colorectal liver metastases at a single centre in South Africa: A report of the ethics and regulatory approval process
}

\author{
H R Etheredge, ${ }^{1}$ MSc Med, PhD; J Botha, ${ }^{2}$ MB BCh, FCS; P Cleaton-Jones, ${ }^{3}$ MB BCh, PhD, DSc, FCD \\ ${ }^{1}$ Wits Donald Gordon Medical Centre, and Wits Human Research Ethics Committee (Medical), University of the Witwatersrand, Johannesburg, \\ South Africa \\ ${ }^{2}$ Transplant Programme, Wits Donald Gordon Medical Centre, and Department of Surgery, University of the Witwatersrand, Johannesburg, South Africa \\ ${ }^{3}$ Steve Biko Centre for Bioethics, and Wits Human Research Ethics Committee (Medical), University of the Witwatersrand, Johannesburg, South Africa
}

Corresponding author: H Etheredge (harriet.etheredge@mediclinic.co.za)

\begin{abstract}
Non-resectable colorectal liver metastases (CLMs) are generally considered an absolute contraindication for liver transplantation. However, a 2013 Norwegian study transplanted livers in 21 patients with CLMs and reported excellent outcomes. The current article reports on the deliberations of the Wits Human Research Ethics Committee (Medical), which resulted in approval of the first liver transplant for a patient with CLMs in South Africa (SA). Factors considered included the scarcity of donor organs in SA, weighing the best interests of different groups of patients and the requirement for extensive and careful information provision. Originally under the auspices of Section 37 of the World Medical Association Declaration of Helsinki (2013), the protocol has now been formalised as a research project.
\end{abstract}

S Afr J Bioethics Law 2017;10(1):5-7. DOI:10.7196/SAJBL.2017.v10i1.534

Non-resectable colorectal liver metastases (CLMs) are generally considered an absolute contraindication for liver transplantation, because of poor outcomes reported during the 1990s. ${ }^{[1]}$ However, a 2013 Norwegian pilot study by Hagness et al. ${ }^{[1]}$ transplanted livers in 21 patients with non-resectable CLMs, and reported excellent oncological outcome and a 60\% 5-year survival. This result far exceeds that which is obtained with chemotherapy. ${ }^{[1]}$ Although a promising development, consensus is that larger studies with longer follow-up times, in different cohorts, are needed to validate the results and to support CLMs as an indication for liver transplant, or not. ${ }^{[2]}$

In South Africa (SA), an upper-middle income country (UMIC), ${ }^{[3]}$ a relatively small number of organ transplants are performed annually. In recent years, teams of committed academic surgeons and physicians have developed, or adopted, new transplant techniques, in a bid to extend this treatment to a larger number of patients. However, the supply of donor organs in SA falls far short of the demand for them. Wits Donald Gordon Medical Centre (WDGMC) is a tertiary academic hospital in Johannesburg, and is one of three hospitals in SA offering liver transplantation. As it falls within the University of the Witwatersrand (Wits) hospital complex, all research taking place at WDGMC requires approval from the Wits Human Research Ethics Committee (Medical) (HREC(M)).

This article reports on the deliberations of the Wits HREC(M) that eventually led to the approval of a research project to transplant, and prospectively follow-up, patients presenting with CLMs at WDGMC. To date, to the best of our knowledge, Columbia is the only other UMIC that has performed a transplant on a patient presenting with $\mathrm{CLMs}^{[4]}$ Both the Norwegian and Columbian articles recorded ethics committee approvals, but no details were mentioned. ${ }^{[1,4]}$
Given that a number of other UMICs, including Belarus, Brazil, China, Iran and Romania host liver transplant programmes, it is foreseeable that they may consider similar research. We hope that this article may be useful for HRECs at other SA transplant centres and in UMICs that are similar to $S A$, where transplant indications and outcomes may differ from those in high-income countries.

\section{The case}

In early 2015, we were approached by Patient A, a well-informed medical doctor diagnosed with non-resectable CLMs. Patient A, who had searched the internet for information, requested consideration for liver transplantation, based on the outcome of Hagness et al. ${ }^{[1]}$ and because he fitted the inclusion criteria. The WDGMC Clinical Research Office advised the transplant team, and forwarded an application to undertake the liver transplant as a case study to the HREC(M). The request was urgent as Patient $A$ required transplant work-up, so the matter was debated by the four co-chairs of the HREC(M), in collaboration with the WDGMC team. All decisions were subsequently ratified by the full HREC(M) (ref. nos. M150362 and M151037). The application was made under section 37 of the 2013 Declaration of Helsinki. ${ }^{[5]}$ This has been the basis for a number of international studies proposing to use new or non-indicated modalities in patients who have exhausted conventional management options, and states:

In the treatment of an individual patient, where proven interventions do not exist or other known interventions have been ineffective, the physician, after seeking expert advice, with informed consent from the patient or a legally authorised representative, may use an unproven intervention if in the physician's judgement it offers hope of saving life, re-establishing health or alleviating 
suffering. This intervention should subsequently be made the object of research, designed to evaluate its safety and efficacy. In all cases, new information must be recorded and, where appropriate, made publicly available. ${ }^{\prime[5]}$

The HREC(M) felt that these terms were sufficient for approving the case, with the condition that the principal investigator report back and formalise the intervention as a research study, if applicable. In making this decision, several points of ethics were deliberated.

\section{Scarcity of donor organs in SA}

Studies using scarce resources (such as donor livers) to treat a condition for which such management is not yet indicated pose unique ethical challenges. Hagness et al. ${ }^{[1]}$ justified their study based on a surplus of donor livers in Norway. In SA, the ethical challenges are heightened, as the supply of donor organs falls short of the demand for them. Donor organs are usually allocated to listed individuals on the basis of need ('sickest person first' principles), and depending on the size of graft required and blood group compatibility.

Because the waiting list for liver transplants in SA is relatively short, the $\operatorname{HREC}(M)$ concluded that it would be ethically sound to allocate a marginal (expanded criteria) graft - which had been refused by all other centres countrywide - to Patient A. A marginal graft would not usually be suitable for implantation, owing to factors such as markedly elevated liver function tests, older age or HIV-positive status of the donor or excessive macrosteatosis on hepatic biopsy. This decision may have been different if the liver waiting list was much longer, with other patients who could have benefitted from a marginal graft - as is often the case with patients who require kidney transplantation.

There was no comparable precedent from other UMICs for the decision to allow a marginal graft for Patient $A$ at the time. In the more recent case of a liver transplant for CLMs in Columbia, it is unclear whether the graft was marginal (the publication states only that the graft was from a 22-year-old male in cold ischaemia for 7.5 hours). It is possible that the Columbian centre had a surplus of donor livers ${ }^{[4]}$ Until the procedure is validated by further research, it seems that offering patients with CLMs marginal grafts is the only ethical solution in the SA context of organ scarcity.

\section{Patients already listed for liver transplants}

A number of patients with recognised indications are waiting for liver transplants at any given time. Because liver transplantation is not accepted for CLMs, it was essential that these patients were not disadvantaged by the decision to perform the transplant on Patient A. Recognising the imperative to balance the best interests of listed patients with the best interests of Patient A, the HREC(M) stipulated that Patient A may receive a donor liver only if there was no other suitable recipient for the graft, countrywide. This decision effectively meant that irrespective of model for end-stage liver disease (MELD) score or urgency, Patient A would not be prioritised for transplant.

\section{Risks and benefits to the patient}

Careful analysis concluded that for Patient A, the anticipated benefits of transplant (prolonged life, possibly by several years) categorically outweighed the concomitant risks (contracting HIV, life-long medication, rejection). The fact that Patient $A$ was a medical doctor and perhaps better able to appreciate the extent of the risk was also a consideration for informed consent. Nonetheless, as per SA ethics guidelines, ${ }^{[6]}$ HREC(M) stipulated that Patient A should be fully informed of the potential risks involved in receiving a marginal graft, and their consequences.

\section{Questions of cost}

Identifying who will bear the financial costs of clinical research is essential for HRECs. SA ethics regulations stipulate that no research costs should be incurred by a participating institution. ${ }^{[6]}$ Liver transplantation is very expensive, and the HREC agreed that either Patient A's medical scheme should fund the transplant on an ad hoc basis, or the patient would need to pay his own medical expenses. In the latter case, the HREC stipulated that Patient A must be notified of all potential costs, in order to make an informed decision. Ultimately, the research procedure was authorised by the medical scheme.

\section{Brief concluding remarks and study progress}

Patient A received a transplant from a 75-year-old donor in August 2015. Based on encouraging short-term findings, a submission was made to the HREC(M) to formalise the protocol as a research study, and the request was approved. Recruitment for the study is ongoing, and the study process is illustrated in Fig. 1.

The mandates to further evidence-based medicine (EBM) on the one hand, and protect research participants on the other, can present HRECs with something of a catch 22 - exemplified by this case. EBM is constantly evolving, as studies identify superior treatment modalities; however, these studies may often seem precipitous at the outset, with too many risks to participants. Nonetheless, it is essential that they take place in order for EBM to evolve. This may require difficult decisions, which are not always widely acceptable. There are a

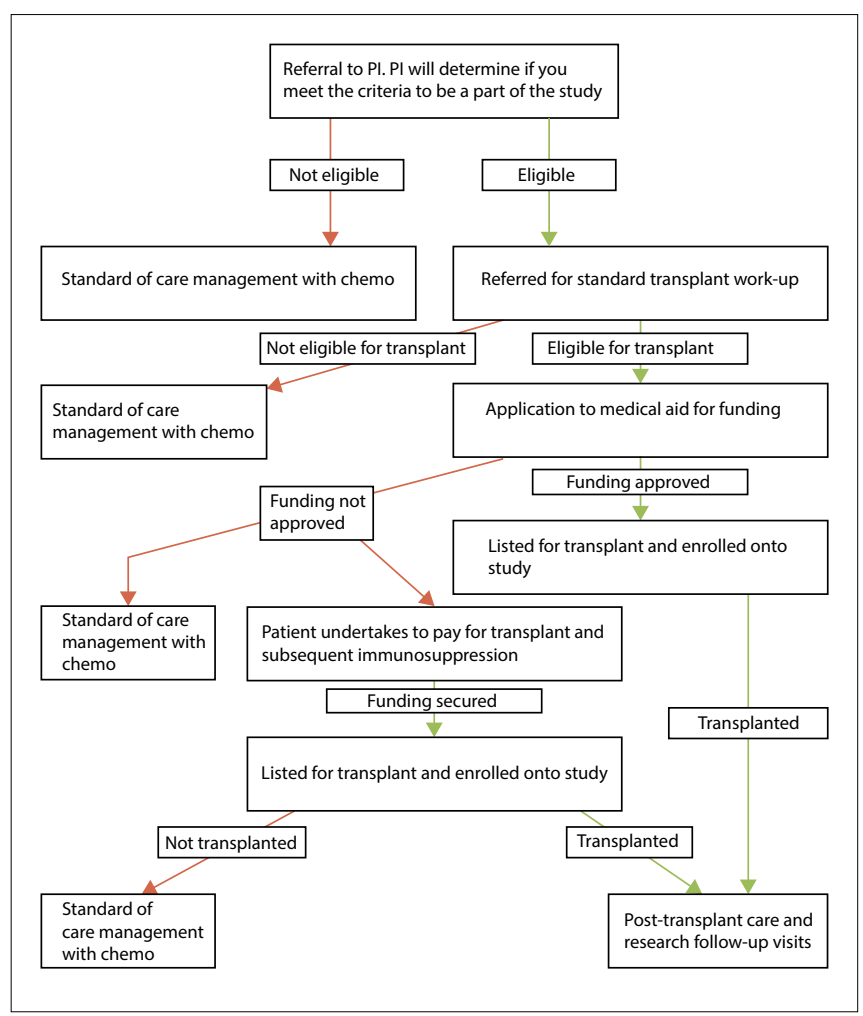

Fig. 1. Study process. 
number of examples of evolving EBM in liver transplant research. Studies of transplant patients with CLMs may follow the same pattern, and should be judiciously encouraged. Primary hepatocellular carcinoma is now considered an indication for liver transplant because of improved screening and the development of standardised criteria (Milan) that have facilitated better outcomes. The same goes for cholangiocarcinoma, with $\sim 65 \%$ of patients recurrence-free after 5 years, having undergone improved neoadjuvant therapy followed by liver transplant. ${ }^{[2]}$

For additional references, please contact the corresponding author.

1. Hagness $M$, Foss $A$, Line $P-D$, et al. Liver transplantation for nonresectable liver metastases from colorectal cancer. Ann Surg 2013;257(5):800-806. http://dx.doi. org/10.1097/SLA.0b013e3182823957
2. Martins PNA, Movahedi B, Bozorgzadeh A. Liver transplantation for unresectable colorectal cancer liver metastases: A paradigm change? Ann Surg 2015;62(1):e12. http://dx.doi.org/10.1097/SLA.0000000000000483

3. The World Bank. World Bank Country and Lending Groups. 2016. Washington DC: World Bank, 2017. https://datahelpdesk.worldbank.org/knowledgebase/ articles/906519-world-bank-country-and-lending-groups (accessed 3 March 2017).

4. Caicedo L, Buitrago D. Thomas $L$, et al. Liver transplantation for unresectable metastases from colon adenocarcinoma. Case Rep Gastroenterol 2017;10(3):808813. http://dx.doi.org/10.1159/000454984

5. World Medical Association. WMA Declaration of Helsinki - Ethical Principles for Medical Research Involving Human Subjects 2013. Geneva: WMA, 2013. http:// www.wma.net/en/30publications/10policies/b3/ (accessed 3 March 2017).

6. National Department of Health, Republic of South Africa. Ethics in Health Research - Principles, Processes and Structures, 2nd ed. Pretoria: National Health Research Ethics Committee. http://www.nhrec.org.za/docs/Documents/ EthicsHealthResearchFinalAused.pdf (accessed 3 March 2017). 